\title{
RESISTÊNCIA DE Eucalyptus globulus E Eucalyptus nitens À FERRUGEM (Puccinia psidii) $^{1}$
}

\author{
Adelica Aparecida Xavier ${ }^{2}$, Eugênio Von. Sanfuentes ${ }^{3}$, Davi Theodoro Junghans ${ }^{4}$ e Acelino Couto Alfenas ${ }^{5}$
}

\begin{abstract}
RESUMO - Avaliou-se a resistência das espécies de Eucalyptus globulus e Eucalyptus nitens inoculadas com um isolado uredinospórico monopustular de Puccinia psidii origininário de plantio de Eucalypstus grandis (UFV-2) em Itapetininga, SP. A avaliação foi realizada aos 12 dias após a inoculação, e quantificou-se a doença por meio de uma escala de notas com quatro classes de severidade da doença (S0, S1, S2 e S3). Em média, aproximadamente $60 \%$ das plantas de E. globulus e $50 \%$ de $E$. nitens foram resistentes a $P$. psidii. A variabilidade intra-específica nos materiais estudados indica ser possível a clonagem de genótipos resistentes para plantio comercial ou para uso em programas de melhoramento genético.
\end{abstract}

Palavras-chave: Controle, ferrugem do eucalipto e variabilidade.

\section{RESISTANCE OF Eucalyptus globulus AND E. nitens TO RUST}

\begin{abstract}
Eucalyptus globulus and Eucalyptus nitens were evaluated for resistance to rust caused by Puccinia psidii. Seedlings were inoculated with a single urediniosporic pustule isolate of P. psidii (UFV-2) obtained from $\boldsymbol{E}$. grandis from Itapetininga, SP. Disease assessment was carried out 12 days after inoculation based on a rust rating scale with four class of severity (SO, S1, S2 and S3). Percentages of resistant plants were $60 \%$ and $50 \%$ for $\boldsymbol{E}$. globulus and $\boldsymbol{E}$. nitens, respectively. The high intra-specific variability found in this study allows using the clonal propagation of resistant genotypes in commercial plantations or in breeding programs.
\end{abstract}

Keywords: Rust control, Eucalyptus disease and variability.

\section{INTRODUÇÃO}

A ferrugem, causada por Puccinia psidii Winter, constitui uma doença potencialmente importante para a eucaliptocultura mundial (COUTINHO et al., 1998), sobretudo para a Austrália, centro de origem do eucalipto, e África do Sul, relevante país eucaliptocultor, onde a doença ainda não foi encontrada.

No Brasil, essa ferrugem constitui uma das principais doenças da cultura do eucalipto (ALFENAS et al., 1989; FERREIRA, 1989; ALFENAS et al., 1997). A partir da década de 1970, com a expansão da eucaliptocultura no país, tornaram-se freqüentes os relatos de surtos de ferrugem. Assim, tornou-se necessário conhecer o comportamento das espécies e procedências introduzidas no Brasil, com objetivo de evitar plantios em larga escala de procedências muito suscetíveis, dado o potencial de danos causados pela doença, principalmente na fase de muda em viveiro e até aproximadamente os dois primeiros anos de plantio (FERREIRA, 1989). Grande variabilidade genética quanto à resistência à ferrugem tem sido encontrada entre espécies e procedências e mesmo entre indivíduos de mesma procedência ou progênie (CARVALHO et al., 1998). Entre as espécies mais suscetíveis, destacamse Eucalyptus grandis W. Hill ex Maiden, E. cloeziana

\footnotetext{
${ }^{1}$ Recebido em 25.08.2006 e aceito para publicação em 29.03.2007

${ }^{2}$ Departamento de Agronomia/CCET - UNIMONTES/ Janaúba-MG. E-mail: <adelica@ unimontes.br>.

${ }^{3}$ Departamento de Silvicultura, Facultad de Ciencias Forestales, Universidad de Concepción, Concepción, Chile.

${ }^{4}$ EMBRAPA/CNPMF- Cruz Das Alma, BA.

${ }^{5}$ Departamento de Fitopatologia da Universidade Federal de Viçosa, Viçosa, MG. E-mail: <aalfenas@ufv.br>.
} 
e E. phaeotricha, sendo a procedência África do Sul de $E$. grandis uma das mais suscetíveis. Como resistentes, destacam-se as espécies e procedências E. pellita $\mathrm{F}$. Muell - procedência 10966; E. microcorys F. Muell procedência Fabriciano - 766 e E. urophylla S.T. Blake - 10131 e como altamente resistente $E$. pellita F. Muell - Helenvale (DIANESE et al., 1984; FERREIRA, 1989).

As espécies de E. globulus La Bill e E. nitens (Deane \& Maid.) Maid. possuem grande valor comercial pelo alto teor de celulose e baixo teor de lignina e têm sido amplamente cultivadas em regiões de inverno chuvoso e temperaturas relativamente baixas, ocupando cerca de $22 \%$ da área total de Eucalyptus no mundo, sendo muito plantada em Portugal, Chile e Espanha (ELDRIDGE et al., 1994) e Uruguai. No Brasil, essas espécies têm sido hibridizadas com outras climaticamente mais adaptadas, visando aumentar o rendimento de celulose e reduzir o teor de lignina. Entretanto, considerando o potencial de plantio desses híbridos em regiões de clima favorável à ferrugem (RUIZ et al., 1989a) e a inexistência de dados sobre a reação de E. globulus e E. nitens a P. psidii, é importante conhecer a resposta de resistência dessas duas espécies à doença, a fim de embasar a seleção de genótipos resistentes para hibridização. Assim, este estudo teve como objetivo avaliar a resposta de resistência de duas espécies de eucaliptos a $P$. psidii sob condições controladas.

\section{MATERIAL E MÉTODOS}

Foram utilizadas mudas obtidas a partir de sementes de E. globulus procedentes das regiões de Quirihue, San Pedro de Ribue, Mariman e Tirua e de E. nitens, de Malloga e Santa Luzia, no Chile. Como padrão de suscetibilidade, utilizaram-se mudas oriundas de sementes de E. grandis procedentes da África do Sul.

As inoculações foram realizadas 60 dias após o transplantio, utilizando-se o isolado urediniospórico monopustular UFV-2 de $P$. psidii, obtido de $E$. grandis procedente de Itapetininga, SP. Esse isolado foi multiplicado em mudas de jambeiro (Sygyzium jambos (L.) Alston), e aos 12 dias após a inoculação (d.a.i.) os uredinióporos foram dispersos em água + Tween 20 a $0,05 \%$. A suspensão foi ajustada para $2 \times 10^{4}$ esporos/ mL e atomizada homogeneamente na superfície adaxial das quatro folhas mais jovens de 50 plantas de cada uma das espécies de Eucalyptus spp. estudadas. Após a inoculação, as mudas foram mantidas em câmara de nevoeiro por $24 \mathrm{~h}$, no escuro, a $25^{\circ} \mathrm{C}$, sendo posteriormente mantidas a $22 \pm 1^{\circ} \mathrm{C}$, com fotoperíodo de 12 h (RUIZ et al., 1989a,b) e intensidade luminosa de $36 \mathrm{mmol} \cdot \mathrm{m}^{-2} \mathrm{~s}^{-1}$. A avaliação foi realizada aos 12 dias após a inoculação, utilizando-se a escala de notas de severidade da doença, conforme Junghans et al. (2003) (Figura 1).

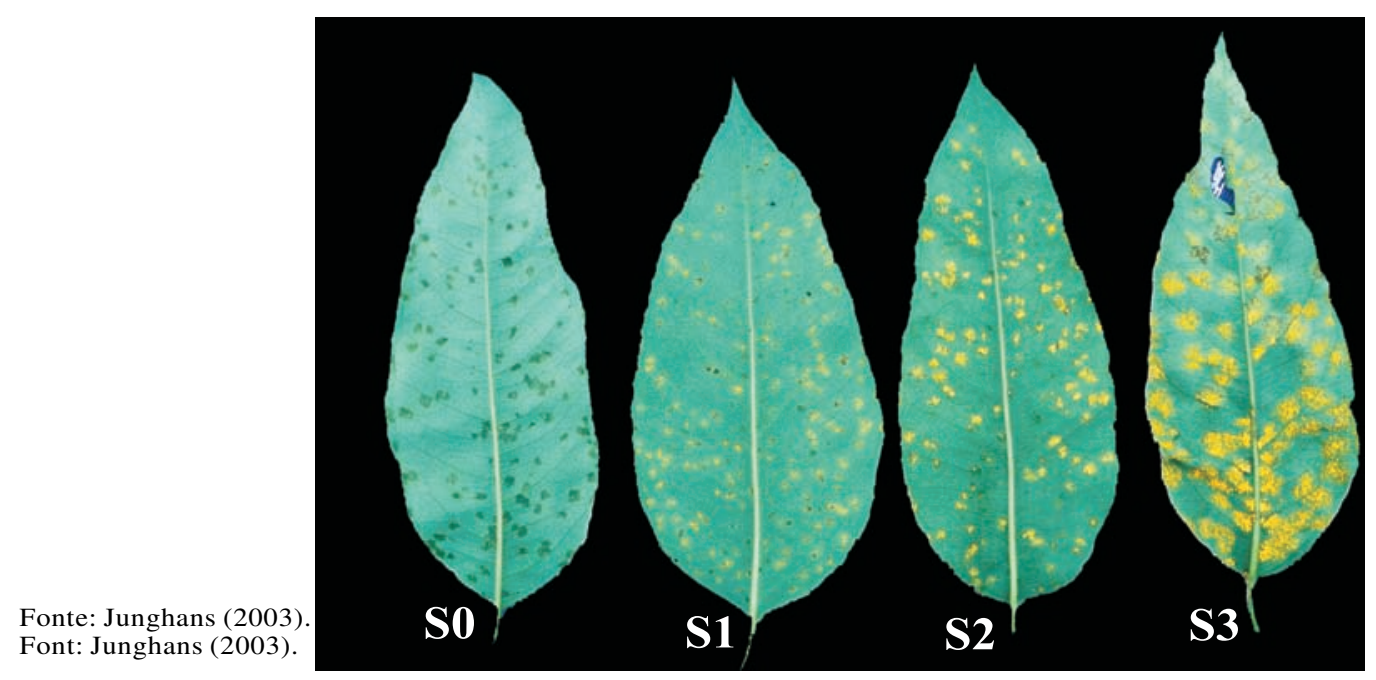

Figura 1 - Escala de notas para avaliação da resistência do eucalipto, com quatro classes de severidade: S0 = imunidade ou reação de hipersensibilidade do tipo "fleck" ou necrótico; $\mathrm{S} 1$ = pústulas < 0,8 mm de diâmetro; S2 = pústulas de 0,8 a 1,6 de $\mathrm{mm}$ de diâmetro; e S3 = pústulas $1,6 \mathrm{~mm}$ de diâmetro.

Figure 1 -Eucalyptus rust severity scale: $S O=$ immunity or hypersensitive reaction (fleck or necrosis type); $S 1=$ pustules $<0.8 \mathrm{~mm}$ diameter; $S 2=$ pustules from $0.8-1.6 \mathrm{~mm}$ diameter; $S 3=$ pustules $>1.6 \mathrm{~mm}$ diameter .

R. Árvore, Viçosa-MG, v.31, n.4, p.731-735, 2007

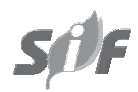


O delineamento estatístico foi inteiramente casualizado, e cada planta constituiu uma unidade experimental. Para cada espécie, avaliaram-se 50 plantas. O ensaio foi repetido duas vezes no tempo. Os números médios de plantas obtidos em cada classe de severidade, nos dois ensaios, foram transformados em porcentagem e apresentados para cada espécie. Esses dados foram previamente transformados para $\sqrt{x+0,5}$ e submetidos à análise de variância $(\mathrm{P}<0,05)$, comparando-se as médias pelo teste de Tukey (5\%).

\section{RESULTADOS E DISCUSSÃO}

Constatou-se variabilidade nas duas espécies avaliadas, bem como entre as procedências dentro de cada espécie, havendo plantas com graus de severidade S0 (grau mínimo) a S3 (grau máximo). Todas as procedências avaliadas foram estatisticamente diferentes de E. grandis (padrão de suscetibilidade). A procedência de E. globulus Quirihue destacou-se entre os demais tratamentos, apresentando menor valor de severidade na escala de notas (Figura 2). As demais procedências de E. globulus e E. nitens Malloga não foram estatisticamente diferentes entre si, porém quando se avalia a relação dos indivíduos resistentes $(\mathrm{S} 0+\mathrm{S} 1)$ e suscetíveis $(\mathrm{S} 2+\mathrm{S} 3)$, observou-se que acima de $72 \%$ foram classificados como resistentes dentro da procedência E. globulus San Pedro de Ribue e E. globulus Marimam e 55\% dentro das procedências de E. globulus Tirua e E. nitens Malloga, evidenciando-se a variabilidade intra-específica presente nessas procedências. Para E. globulus, a procedência Tirua destacou-se como a mais suscetível, com $41 \%$ das plantas nas classes de severidade de S2 e S3 (Quadro 1). Estatisticamente, as plantas de E. nitens procedência Santa Luzia apresentaram resposta intemediária ao comportamento de $E$. grandis e às demais procedência avaliadas, exceto E. globulus Quirihue (Figura 2). As procedências de E. nitens avaliadas exibiram menor número de plantas na classe S0 (resistência completa), com uma proporção de 1:1 entre indivíduos resistentes (S0+S1) e suscetíveis (S2+S3), entretanto os números de indivíduos suscetíveis foram $34 \%$ menores quando comparados os de E. grandis - África do Sul (Quadro 1). Nas procedências Quirihue, San Pedro de Ribue e Mariman, o número de plantas que apresentaram resistência completa (S0) caracterizada pela imunidade e HR com necrose ou "fleck" foi aproximadamente $100 \%$ maior que aqueles apresentados nas procedências das espécies de E. nitens e E. grandis (Quadro 1). Essa reação em eucalipto caracteriza a restrição do desenvolvimento do patógeno no sítio de infecção logo após a formação do primeiro haustório (XAVIER et al., 2002) e a expressão de poucos genes em resposta à presença do patógeno, caracterizando uma resistência do tipo vertical (AGRIOS, 1997). Esse tipo de resistência observada nessas espécies é uma característica desejável para a produção de mudas e seleção de indivíduos para serem utilizados no melhoramento.

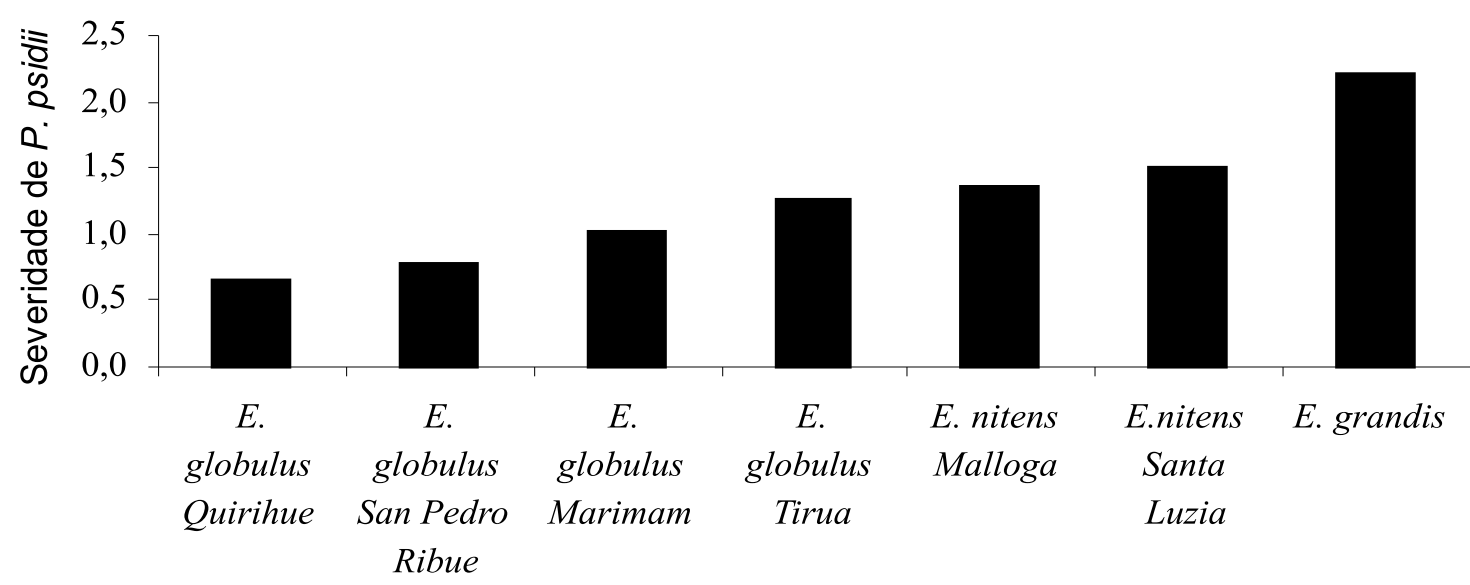

\section{Procedências}

Figura 2 - Médias de notas de severidade de $P$. psidii obtidas pela escala de Junghans et al. (2003) em quatro procedências de Eucalyptus globulus, duas de Eucalyptus nitens e Eucalyptus grandis.

Figure 2 - Rust severity scores obtained from Junghans et al. (2003) scale on four provenances of Eucalyptus globulus, three Eucalyptus nitens, and one Eucalyptus grandis. 
Quadro 1 - Severidade da ferrugem em mudas seminais de E. globulus e E. nitens provenientes de diferentes regiões produtoras de sementes do Chile, comparadas com E. grandis (procedente da África do Sul), inoculadas artificialmente com o isolado monopustular UFV-2 de P. psidii

Table 1 -Rust severity on seedlings of E. globulus, E. nitens and E. grandis, Chilean provenances, compared to E. grandis, South African provenance, inoculated a single pustule of P. psidii (UFV-2)

\begin{tabular}{ccccccc}
\hline Espécie/origem das sementes & \multicolumn{5}{c}{ Freqüência (\%) de plantas } \\
\cline { 2 - 7 } & S0 & S1 & S2 & S3 & R (S0+S1) & S (S2+S1) \\
\hline Eucalyptus globulus & & & & & \\
Quirihue & 76,47 & 1,96 & 9,80 & 11,76 & 78,43 & 21,56 \\
San Pedro de Ribue & 58,93 & 14,28 & 17,68 & 8,93 & 73,21 & 26,61 \\
$\quad$ Mariman & 51,79 & 14,29 & 14,29 & 19,26 & 72,08 & 33,55 \\
$\quad$ Tirua & 41,18 & 17,65 & 17,65 & 23,53 & 58,83 & 41,18 \\
Eucalyptus nitens & & & & & & \\
$\quad$ Malloga & 25,53 & 29,79 & 14,89 & 29,79 & 55,83 & 44,68 \\
Santa Luzia & 27,87 & 22,95 & 16,39 & 32,79 & 50,82 & 49,18 \\
Eucalyptus grandis & 4,08 & 10,20 & 38,78 & 46,94 & 14,28 & 85,72 \\
\hline
\end{tabular}

Neste trabalho, entretanto,utilizou-se apenas um isolado de $P$. psidii e, diante da variabilidade do patógeno demonstrada por diversos autores (CASTRO et al., 1983; COELHO, 1988; APARECIDO, 2001; XAVIER, 2002), seria interessante que as avaliações para tais propósitos utilizassem isolados de $P$. psidii obtidos da Região Sul, já que pode existir dentro da população do patógeno isolados mais adaptados às condições dessa região. Inoculações de isolados obtidos de diferentes plantas hospedeiras dessa região demonstraram que a virulência desses isolados variou nas diferentes plantas hospedeiras dentro da família Myrtaceae, assim como a severidade em diferentes clones de $E$. grandis testados (XAVIER, 2002). Isso demonstra a existência de variabilidade genética da população de $P$. psidii nessa região e torna importante a seleção para resistência a esse patógeno para o melhoramento visando à resistência a doenças.

A variabilidade intra-específica para resistência observada neste trabalho também tem sido verificada por diversos autores, com outras espécies e procedências de Eucalyptus spp. (CARVALHO et al., 1998; DIANESE et al., 1984; FERREIRA e SILVA, 1982). A padronização de metodologia tanto da inoculação quanto da avaliação é importante para discriminar diferenças entre os materiais testados. Neste estudo, marcaram-se folhas com mesma idade fenológica para serem avaliadas, pois, conforme determinado por Xavier (1997), a severidade da doença pode variar em função da sua idade. Além disso, com a utilização da escala desenvolvida por Junghans
(2003) foi possível diferenciar graus de resistência nas duas espécies e procedências avaliadas. Assim, a seleção de genótipos resistentes à ferrugem poderá fornecer plantas resistentes para clonagem e plantio comercial no sul do país, dada à adaptação das duas espécies a regiões frias com chuvas de inverno. Essas informações são também importantes para os programas de melhoramento genético que visam à obtenção de híbridos interespecíficos, envolvendo cruzamentos dessas duas espécies com materiais mais adaptados às condições de clima do Brasil. Todavia, é recomendável que a seleção dos materiais de interesse ( $\mathrm{S} 0$ e S1) seja realizada por meio de inoculações artificiais sob condições controladas, para evitar a seleção involuntária de plantas-escape.

As espécies de E. globulus e E. nitens mostraram variabilidade intra-específica para resistência à ferrugem. E. globulus exibiu maior porcentagem de indivíduos resistentes que E. nitens. A identificação de materiais resistentes à ferrugem nessas espécies deve ser uma das primeiras etapas dos programas de plantações com híbridos clonais.

\section{AGRADECIMENTOS}

Ao pesquisador Edval Ângelo Valverde Zauza, pelas sugestões e pela revisão do manuscrito.

\section{REFERÊNCIAS}

ALFENAS, A. C.; DEMUNER, N. L.; BARBOSA, M. M. A ferrugem e as opções de controle. Correio Agrícola, n.1, p.18-20, 1989. 
ALFENAS, A. C. et al. Eucalyptus rust: genetic variability of elite clones and histological characterization of the resistance reaction.

IUFRO CONFERENCE ON SILVICULTURE ON IMPROVEMENT OF

EUCALYPTUS, Colombo, 1997. PROCEEDINGS... Colombo: Embrapa Centro Nacional de Pesquisas Florestais, 1197.v.2. p.60-64.

AGRIOS, G. Plant pathology. 4 ed. London: Academic Press, 1997. 635p.

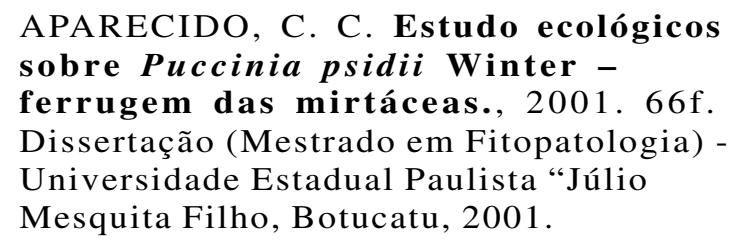

COELHO, L. Variabilidade fisiológica de Puccinia psidii Winter - ferrugem do eucalipto. 1988. 68f. Dissertação (Mestrado em Fitopatologia) - Universidade Federal de Viçosa, Viçosa, MG, 1988.

CARVAlHO, A. O. et al. Resistência de espécies, progênies e procedências de Eucalyptus à ferrugem, causada por Puccinia psidii Winter. Pesquisa Agropecuária Brasileira, v.33, n.2, p.139-147, 1998 .

CASTRO, H. A. et al. Inoculação cruzada de Eucalyptus, goiaba (Psidium guajava) e jambeiro (Syzygium jambos) com Puccinia psidii. Fitopatologia Brasileira, v.8, p.491-497, 1983.

COUTINHO, T. A. et al. Eucalyptus rust: a disease with the potential for serious international implications. Plant Disease, v.82, n.7, p.819-925, 1998.

DIANESE, J. C.; MORAES, A. T. S.; SILVA, A. R. Response of Eucalyptus species to field infection by Puccinia psidii. Plant Disease, v.68, n.4, p.314-316, 1984.
ELDRIDGE, K. et al. Eucalypt

domestication and breeding. Oxford:

Clarendon Press, 1994. 288p.

FERREIRA, F. A. Ferrugem do eucalipto. In: Patologia florestal - principais doenças florestais no Brasil. Viçosa, MG: Sociedade de Investigações Florestais/Universidade Federal de Viçosa, 1989. p.129-146.

FERREIRA, F. A.; SILVA, A. R. Comportamento de procedências de Eucalyptus grandis e de E. saligna à ferrugem (Puccinia psidii). Fitopatologia Brasileira, v. 7, n.1, p.23-28, 1982.

JUNGHANS, D. T.; ALFENAS, A. C.; MAFFIA, L. A. Escala de notas para quantificação da ferrugem em Eucalyptus. Fitopatologia Brasileira, v.28, n.2, p.184-188, 2003.

RUIZ R., A. R. et al. Influência da temperatura, do tempo de molhamento foliar, fotoperíodo e intensidade de luz sobre a infecção de Puccinia psidii em eucalipto. Fitopatologia

Brasileira, v. 14, n.2, p.55-61, 1989a.

RUIZ, R. A. R.; ALFENAS, A. C.; FERREIRA, F. A. Influência de temperatura, luz e origem do inóculo sobre a produção de uredosporos e teliosporos de Puccinia psidii. Fitopatologia Brasileira, v. 14, n.2, p.70-73, 1989 b.

XAVIER, A. A. Histoptologia da interação Puccinia psidii e virulência de isolados do patógeno em espécies de Myrtaceae. 2002. 71f. Tese (Doutorado em Fitopatologia) Universidade Federal de Viçosa, Viçosa, MG, 2002.

XAVIER, A. A. Patogênese da ferrugem (Puccinia psidii) em folhas e genótipos suscetíveis e resistentes de Eucalyptus grandis. 1997. 112f. Dissertação (Mestrado em Fitopatologia) - Universidade Federal de Viçosa, Viçosa, MG, 1997.

XAVIER, A. A. et al. Infection of resistant and susceptible Eucalyptus grandis genotypes by urediniosporos of Puccinia psidii. Australasian Plant Pathology, v.30, p.277-281, 2001. 
